\title{
The Community Solvency Margin for Insurance other than Life Insurance : Instrument of Fair Competition or a Source of Disparities among Firms ?
}

(English summary)

\section{Introduction}

\subsection{Objective of the study}

"Insurance is an international industry with specific national characteristics. The introduction of a minimum solvency margin on the basis of common general regulations within the European Community provides an element of competitive equality" 1 . It constitutes at least a formal equality of treatment which should be evaluated for its effects on the equality of opportunity among firms on both the national and international levels.

The question is all the more important since the texts of the regulations permit some interpretation by national supervisory authorities and allow them a margin of manoeuvre. Disparities will exist here so long as harmonization of implementation by different countries is not achieved.

The study of the impact of the solvency margin on the equality of opportunity for companies to enter the business of insurance, other than life insurance, and to maintain their position there involves starting from differences in regulations or their implementation and subsequently arriving at their economic consequences.

The general problem so defined, we wish to state here that our intention is not to call into question statutory and legal provisions, but rather to study their possible repercussions on competition among companies. Whatever negative comments may eventually be made, the good reasons for financial supervision in general, and for the statutory solvency margin in particular, will not be called into question. The latter is the foundation stone of a new order which, after twenty years of reflection and negociation, commands respect. Moreover, general economic conditions may well have changed in this respect and the differences between countries may have deepened. Sometimes these changes and accentuations are advanced to query the principle of common regulations : for our part, we will appraise them only in so far as they affect the subject deliberately chosen for this study, namely the changes in the conditions of competition among firms attributable to the introduction of the Community solvency margin for insurance other than life insurance.

\subsection{Competition and disparities among firms}

"Competition" does not always have the same meaning for the head of a firm and for the economist. Faced with new legal provisions, an insurance company is

1 PLESCOFF, Georges: "Address on the occasion of the 25th anniversary of the European Insurance Committee", October 1978. 
concerned that the rules of the game to gain access to markets or maintain a position there do not change to its disadvantage. Any statutory modifications in this direction are felt as distorsions in the conditions of competition. The economist will think differently if the ensuring selection of firms is such as to improve the optimal supply to the market lastingly and thus to contribute to overall growth (a modern idea of competition, especially the new theory of John M. Clark ${ }^{2}$ ). This will be particularly on the supply side and to the protection of the consumer.

The obligation (i) to take into account accumulated profits and (ii) to make profits and hold them at levels established by law year after year (essential characteristics of the margin) represents, for several of the countries studied, an important modification of their framework of regulations. This dual constraint or that, which it implies, of perhaps resorting more frequently to the capital market, is going to restrict the business activities of some insurance companies and be seen as a factor limiting market access. The economist will not necessarily consider these obstacles as distortions to the conditions of competition; being of the opinion that by tending to abolish dumping rates and by eliminating firms with high take-over and administrative costs the margin can contribute to the optimal supply of these markets. Mergers which may arise from the introduction of the margin are not necessarily incompatible with the interests and protection of insured parties.

However it will be admitted that the new solvency requirements may well, a priori, modify the conditions of competition among suppliers and that firms could be affected in different degrees according to their characteristics (size, legal status, location of head office, etc.). Disparities among companies arising from the introduction of the margin (henceforth called singly "disparities") will be discussed.

The present study aims at identifying precisely these disparities and their possible implications. Their positive or negative effects on competition in real terms must be evaluated later by observing economic facts (rather than by using predictions hardly compatible with the rigorous analysis used in economic research).

\subsection{Characteristic types of disparities}

The obstacles which an insurance company may meet following the introduction of the margin - in practice since 1979 on the basis of annual accounts to 31 December 1978 - arise from possible difficulties it may encounter :

(i) in giving proof of the sufficient level of its own funds (static aspect of the margin);

(ii) in financing the margin necessary for business expansion (dynamic aspect).

In the first case, the level of its own assets which the company must declare apart from the common rules for calculation - depends on different bases of reference according to the location of the head-office (within the Community or elsewhere).

2 On this subject see SCHMIDT, Reimer: "The importance of the financial strength of insurance companies with reference to competition, especially on the European market ", in the Report of the Monte-Carlo Rendez-vous, September 1978. This conception of competition diverges from the traditional theory (Augustin Cournot, Jean Marchal, etc.) which implies an immediate response to demand with quantities as large as possible and at the lowest possible price. 
Moreover, for companies operating on the same national territory, the supervisory authorities competent to pronounce on the adequacy of owned assets belong to different member states according to the legal status and domicile involved (head offices of national companies and branch-offices of non-member states / branch offices of Community companies). As to the level of net assets which the company may include in its accounts, this depends on the evaluation of assets and liabilities. This operation creates a problem because the rules for estimating have not been harmonized among member-states, thus leaving some freedom of interpretation to the supervisory authorities of the country competent to pronounce on solvency. Disparities between companies may therefore be present at the static stage of giving proof of the margin.

From the dynamic angle, financing the margin depends on the operational situation of the company and the general economic situation in which it does business. The introduction of the margin does not change these conditions - at least not directly - but they affect the profit-making capacity in various ways (according to the classes of insurance concerned) self-financing (via fiscal policy, general dividend distribution policy), external financing (through national capital markets) and the maintenance of accumulated net profits in real terms (according to the rate of inflation, the possibilities of investment in real values). Existing disparities among insurers under these different heads become more of a handicap once companies are obliged to accumulate high minimum profits and to maintain their real value, as the existence of the margin implies.

Whether the disparities considered be due to static or dynamic features of the margin, it would be necessary to distinguish between :

(i) disparities capable of modifying the conditions of competition among suppliers on the same national market;

(ii) disparities capable of hindering access to the European Community insurance market and the growth of business in third countries.

Moreover a distinction can be made between long and short-term disparities. The former are due to incomplete harmonization of interpretations of the common regulations or general economic conditions. The latter can only be ascribed to the length of time necessary for the different national authorities concerned to establish financial supervision of insurance companies. It is to be noted, for example, that a new bill on insurance in accordance with the Community directives was only tabled in the Dutch Parliament in 1979. In other countries, such as Italy, at the start of 1980 changes in legislation had yet to be completed by the issuing of statutory regulations so that the new law could be fully applied.

The long-term disparities identified will indicate where harmonization is desirable to prevent them becoming permanent. As to the short-term ones, they have been noted principally to avoid confusion with the former; they will serve as references in following future progress in the implementation of the new regime.

Objective disparities should be distinguished from subjective ones. The former are the result of an actual situation, either as regards regulations or general economic conditions. Disparities can be considered subjective when they are the result of the awareness of a situation pre-existing the margin but activated by its introduction or due to company behaviour, well founded perhaps, but not required by the new regime. For 
an example of this second type consider the reservations an insurance company may have, in some countries but not in others, in declaring latent hidden reserves though it is permitted to do so in order to prove its solvency.

To recapitulate, an effort has been made in this study to identify disparities among companies classifiable, a priori, according to the following characteristics :

- disparities in declaring the current margin / in financing the future margin ;

- disparities in growth on a single national market / on foreign markets (in the Community or third countries) ;

- long-term/short-term disparities ;

- subjective / objective disparities.

In practice, these different types of characteristics are combined according to the specific sources of the disparities. In the following chapters disparities among companies will be studied according to their origins, without considering their size. Before proceeding further, a preliminary comment on the coverage of the study will be inserted.

\subsection{Coverage of the study}

Without wishing to overlook problems of competition among firms which arise from the composition of premium portfolios, the size of the firm or taxation, this study emphasizes especially the disparities more strictly linked with the introduction of the margin of which, to our knowledge, are less frequently mentioned.

The disparities studied here have to do with :

- the economic consequences of the uniform calculation of the margin (part 2);

- the principle of supervision and the practice of the solvency certificate (part 3 );

- the taking into account of hidden reserves resulting from the underestimation of assets (part 4) or the overestimation of liabilities (part 5);

- financing the margin (part 6) whether the disparities arise from differences in operating conditions, financing conditions or the general economic situation.

Moreover, the study is limited to the following member countries : the Federal Republic of Germany, Belgium, France, Italy, the Netherlands and the United Kingdom. Problems faced by companies belonging to third countries have been considered, in particular for Switzerland.

\section{Conclusions : Evaluation of disparities among firms arising from the introduction of the solvency margin}

In establishing their solvency margin, firms in the Community find themselves technically in different situations according to the location of their head-office because of the persistance of national controls. But on the whole, the economic consequences of differences in treatment are slight, though German and Belgian firms suffer some disadvantages and French and Italian firms enjoy some advantages. The main reason for the slight significance of the effect of the many disparities noted in the course of this study lies in the fact that, when necessary, all member states permit hidden reserves to stand for the margin, though the position of the Federal Republic of Germany is very conservative. Belgian companies are handicapped by the method 
used for calculating the margin, because it is also levied on that portion of the premium which does not correspond to the insured risks - this is high in Belgium. French companies benefit primarily from a favourable treatment of shortfalls and subsidiary accounts.

At this stage of giving proof of the margin some disadvantages for small and medium companies appear more evident. In addition to the cost structure which often has an adverse effect on the ratio between the claimable margin and the risks insured by small and medium companies, resort to reinsurance is taken into account in such a way that the linked reduction in the margin cannot in practice be less than the rate of the years when reinsurers intervene rarely in settling claims. Big companies which are exposed to smaller fluctuations generally disregard the incidence of the calculation of the coefficient of reduction for reinsurance. Leaving aside these special features, in the last analysis small and medium companies may be considered to enjoy a net advantage when proving the margin because the rate is barely above that of larger companies. The range of investments of the latter, which can mitigate variations in chances of covering their own resources or their underwriting liabilities is a factor among others, which must be borne in mind to avoid concluding, even if true in actuarial terms, that big companies are disadvantaged because they do not benefit from a rate of margin decreasing with respect to their volume of business.

In supplying the margin, the specific circumstances of the insurance sector in each of the countries studied are a far more evident source of disparities among companies than national regulations and practices for financial control. In comparing the effects of the restraints imposed on the management of investments of insurance other than "life" in the different countries studied, the United Kingdom and the Netherlands seem to favour the formation of asset surpluses. In France and Italy on the contrary, circumstances are much less favourable and the Federal Republic of Germany and Belgium occupy an intermediate position (the Belgian situation resembles that of the Netherlands however). Generally speaking, multi-branched companies or those belonging to national or international groups are equally favoured to compensate the restrictive effects arising from the regulations on insurance for damages.

The formation of a budgetary surplus however depends also on the general economic conditions prevailing in each country. From this view point, the Federal Republic of Germany and the United Kingdom offer the most advantages, Belgium and the Netherlands the least.

Combining, on the one hand the following view points : (1) the requirements of solvency control, (2) the conditions for asset surplus formation specific to the insurance field, and (3) general economic conditions which, ultimately, govern the growth and maintenance in real terms of owned resources (and therefore of the margin); and on the other, leaving aside the existing technical and financial circumstances of the various companies operating within the Community, the most favourable conditions for complying with the new statutory requirements on solvency are to be found combined in the Federal Republic of Germany, followed by the United Kingdom. The most critical conditions, on the contrary, seem to be present together in Belgium. The balance of advantages and disadvantages offered at the three levels considered in France and Italy is more difficult to establish: the position of these countries approaches the average and is hardly different from that of the Netherlands. 
Firms belonging to non-member countries operating within the Community see their position somewhat weakened by the new regulations, though according to the country concerned (e.g. Switzerland), the operating conditions and the overall economic situation, favourable on the whole, may compensate for the concomitant drawbacks.

In the last analysis the effects of the growth of a company according to its field of activity, and its membership, or not, in national or international groups appear more important than differences arising from the location of the head-office or the country of operations. To conform with the financial requirements implicit in the Community solvency margin and to continue expanding, multi-branched companies or those connected with groups are better placed than those with a single activity and the firms, often small or medium ranked with them, which had been able to increase their market share rapidly. In short, the most dynamic companies are the most affected, especially if in the past they already used to rely on reinsurance or had exhausted their capacity to seek new capital in the financial market to increase their own resources. It should be remarked however that a healthy development in insurance can only be progressive, and that consequently it is not opportune to dramatize the consequences of the restraint to growth which the Community margin represents.

In the economic sphere, the protection of the insured party which the solvency margin is supposed to strengthen could well be accompanied by a weakening of the most dynamic companies and by some mergers of insurance companies within the Community. Mergers are compatible with the protection of the assured party so long as the elimination of high-cost firms does not open the way to cartel agreements. There is a long way to go from the cases of dumping which were occurring even recently in certain markets to conditions where only a few companies would be able to impose their rates. At present, as can be observed in other sectors of the economy, insurance mergers in Western Europe, accompanied by greater efficiency, would seem to correspond to a desirable trend. Therefore this economic consequence of the solvency margin should not be resisted.

The existence of different conditions governing the field of insurar se for damages in member countries is not necessarily a bad thing either. Within the framework of freedom of establishment already extended within the European Community to insurance activities, it belongs to national governments to judge the interest in keeping headoffices of transnational companies on their territory and to create suitable conditions to this end. The insurance industry is an option in national development strategy just as much as the automobile industry or any other advanced industry. It is for national industries feeling themselves discriminated against at Community and international level to plead their case both with the authorities and with public opinion.

If mergers and planning of the insurance sector in certain member countries can be considered, for both insured parties and insurers, as positive economic repercussions of the introduction of a statutory solvency margin for the European Community as a whole, it would none the less be advisable to avoid that this should result in systematically slowing down the growth of rapidly-expanding smaller companies. The difficulties which the most dynamic of these may meet in maintaining or developing their position could constitute, in the long-term, an attack on the optimal supply of the market. With or without regulations in the field of solvency control (e.g. to remedy the consequences of fluctuations in the share of reinsurers in claim settlements or in fiscal 
matters) the understanding and insight of the supervisory bodies will remain, as in the past, the best safeguards for the balanced development of the sector on a national scale. On a European scale, the recently initiated coordination between supervisory bodies has shown that it can avoid the negative consequences of the most glaring disparities, even if the principle of harmonization (e.g. for hidden capital gains) must yet be translated in practice into an attitude equally understanding and free from all ostracism. 